\title{
Neutrino absorption cross sections in supernova environment
}

\author{
J.M. Sampaio ${ }^{1}$, K. Langanke ${ }^{1}$ and G. Martínez-Pinedo ${ }^{2}$ \\ ${ }^{1}$ Institut for Fysik og Astronomi, Århus Universitet, DK-8000 Århus C, Denmark \\ ${ }^{2}$ Departement für Physik und Astronomie der Universität Basel, Basel, Switzerland
}

(November 28, 2018)

\begin{abstract}
We study charged-current neutrino cross sections on neutronrich nuclei in the mass $A \sim 60$ region. Special attention is paid to environmental effects, i.e. finite temperature and density, on the cross sections. As these effects are largest for small neutrino energies, it is sufficient to study only the GamowTeller (GT) contributions to the cross sections. The relevant GT strength distributions are derived from large-scale shell model calculations. We find that the low-energy cross sections are enhanced at finite temperatures. However, for $\left(\nu_{e}, e^{-}\right)$reactions Pauli blocking of the electrons in the final state makes the cross sections for low-energy neutrinos much smaller than for the competing inelastic scattering on electrons at moderate and large densities. Absorption cross sections for low-energy antineutrinos are strongly enhanced at finite temperatures.
\end{abstract}

PACS numbers: 26.50.+x, 23.40.BW, 21.60Cs 
It has long been recognized that neutrino-induced reactions play a crucial role during the collapse stage of a type II supernova [1]. In particular, elastic neutrino scattering off nuclei reduces the neutrino mean-free path and leads eventually to 'neutrino trapping' at densities in excess of a few $10^{11} \mathrm{~g} / \mathrm{cm}^{3}$. Subsequently the neutrinos in the core are thermalized by inelastic neutrino-electron scattering. Obviously collapse simulations require a detailed description of neutrino transport which is nowadays achieved within Boltzmann neutrino transport calculations and should in principle include all potentially important neutrino reactions [2, [3]. Haxton pointed out that charged- and neutral-current reactions on nuclei should be included in these simulations [4]. Interpreting ${ }^{56} \mathrm{Fe}$ as a representative nucleus the relevant neutrino-nucleus cross sections have been calculated in [5] based on the allowed and first-forbidden ground-state response and compared to other weak-interaction processes under core-contraction conditions.

Neutrino-induced reactions are usually dominated by capture to the giant resonances. As a consequence allowed (Fermi and Gamow-Teller) transitions determine the neutrino cross sections at low energies, while first-forbidden transitions cannot be neglected for neutrino energies in excess of say $20 \mathrm{MeV}$. We also note that for a nucleus with negative $Q$-value, like ${ }^{56} \mathrm{Fe}$, a minimal neutrino energy is required. Consequently the cross section drops sharply at low energies. However, for neutronrich nuclei, as typically encountered during the late core contraction stage, the $Q$-value is positive allowing $\left(\nu_{e}, e^{-}\right)$reactions for all neutrino energies. On the contrast, $\left(\bar{\nu}_{e}, e^{+}\right)$reactions have to overcome the reaction $Q$-value. The picture described here corresponds to neutrino reactions on the nuclear ground state.

Now we will turn our discussion to neutrino reactions on nuclei at finite temperature, described by a Boltzmann distribution of nuclear states. There are giant resonances built on all these excited states and due to Brink's hypothesis the excitation energy of these resonances in the daughter nucleus are the same as for the ground state, only shifted by the excitation energy of the parent state [6]. If Brink's hypothesis is exactly valid, the nuclear response is independent of the initial state and the cross section for the nuclear ground state is the same as at finite temperature. We will demonstrate in the following that this picture, put forward in [5], is actually valid at low temperatures. However, at the temperatures relevant for the core contraction stage $\left(T>10^{10} \mathrm{~K}\right)$ there are states in the thermal ensemble which do not fulfill the Brink hypothesis. These are the giant resonances built on the low-lying daughter states in the inverse reaction. Fuller et al. [7] have noticed the importance of these states for stellar beta-decay (equivalent to the $\left(\nu_{e}, e^{-}\right)$reaction) and have introduced the name 'backresonances' for these states. We will in the following see that, for supernova conditions, the backresonances are more important for $\left(\bar{\nu}_{e}, e^{+}\right)$reactions. The backresonances are expected to have, besides the giant resonance contribution from Brink's hypothesis, an additional component with a sizable nuclear matrix element. Importantly this component is particularly favored by phase space for small neutrino energies.

From this general discussion, which will be supported by our calculations, we expect that it is sufficient to study the finite temperature effects on the Gamow-Teller (GT) contribution to the neutrino-nucleus cross section as i) this component dominates the cross section at low neutrino energies and ii) the GT giant resonances reside at significantly lower excitation energies than those for forbidden responses, i.e. the GT backresonances are thermally more easily accessible than backresonances for other multipoles. We note that there are no Fermi backresonances in $\left(\nu_{e}, e^{-}\right)$reactions, while for $\left(\bar{\nu}_{e}, e^{+}\right)$reactions on neutronrich nuclei these 
states are at too high excitation energies in the parent nucleus to be of importance. For neutrino scattering at low energies it is valid to neglect the dependence of the nuclear multipole operators on momentum transfer.

Restricting ourselves to the GT contribution only, the neutrino-nucleus cross section is given by

$$
\sigma\left(E_{\nu}\right)=\frac{G_{F}^{2} \cos ^{2} \theta_{C}}{\pi} \sum_{i f} \frac{\left(2 J_{i}+1\right) \exp \left[-E_{i} / k T\right]}{G} k_{e}^{i f} E_{e}^{i f} F\left( \pm Z+1, E_{e}^{i f}\right) B_{i f}(G T)
$$

where $G_{F}$ is the Fermi constant, $\theta_{C}$ the Cabibbo angle, and $k_{e}$ and $E_{e}$ the momentum and energy of the outgoing electron or positron. The function $F\left(Z, E_{e}\right)$ corrects for the Coulomb distortion of the outgoing lepton wave function, where the plus sign refers to electrons. The sum is over the final $(f)$ and initial $(i)$ nuclear states, where the latter have excitation energy $E_{i}$ and angular momentum $J_{i}$, and $G$ is the nuclear partition function. Finally, $B_{i f}(G T)$ defines the $B(G T)$ value between the initial and final nuclear states. The nuclear structure information used in this paper (spectra and $B(G T)$ values) are based on large-scale stateof-the-art shell model calculations. These studies are in detail described in Refs. [8,9] which also demonstrate that these shell model studies reproduce the nuclear properties, including the GT strength distributions, quite well.

To understand the relevance of the various components our investigations have been performed in a sequence of steps. To demonstrate the results we have chosen the nucleus ${ }^{56} \mathrm{Fe}$, following [5]. At first we evaluate the cross section (1) by explicitly including the lowest excited states, but neglecting the contributions of the backresonances. Fig. 1a compares the $\left(\nu_{e}, e^{-}\right)$cross section as calculated for the ground state $(i=0)$ with the one obtained by considering the lowest 4 states in ${ }^{56} \mathrm{Fe}$. The study has been performed for the same condition as adopted in Fig. 1a of [5], i.e. $T=10^{10} \mathrm{~K}$ (equivalently $0.86 \mathrm{MeV}$ ). Supporting the argumentation of [5] we find nearly identical results in both approaches, as the Brink hypothesis is well fulfilled for these lowest states (other examples for the validity of this hypothesis are given in [9]).

In the second step we study the influence of the backresonances on the cross section at finite temperature. We will make the assumption that the GT strength distribution $S_{G T}^{i}\left(E_{f}\right)$ for an initial state $i$ can be split into two components $S_{G T}^{i}\left(E_{f}\right)=S_{B r i n k}^{i}\left(E_{f}\right)+S_{b a c k}^{i}\left(E_{f}\right)$ where the first component obeys Brink's hypothesis, i.e. $S_{\text {Brink }}^{0}\left(E_{f}\right)=S_{\text {Brink }}^{i}\left(E_{f}+E_{0}\right)$, and the second component represents the backresonances built on the lowest states in the daughter nucleus. Upon inserting into Eq. (1) the sum over the first component reduces to the ground state contribution. We then have

$$
\begin{aligned}
\sigma\left(E_{\nu}\right)=\frac{G_{F}^{2} \cos ^{2} \theta_{C}}{\pi} & \left\{\sum_{f} k_{e}^{f} E_{e}^{f} F\left( \pm Z+1, E_{e}^{f}\right) B_{0 f}(G T)\right. \\
+ & \left.\sum_{i f} \frac{\left(2 J_{i}+1\right) \exp \left[-E_{i} / k T\right]}{G} k_{e}^{i f} E_{e}^{i f} F\left( \pm Z+1, E_{e}^{i f}\right) B_{\text {back }, i f}(G T)\right\}
\end{aligned}
$$

The nuclear matrix elements for the backresonance part have been derived from large-scale shell model calculations [8] for the lowest (typically 4-12) daughter states in the inverse direction. $\mathrm{B}(\mathrm{GT})$ values, which connect these daughter states to the parent ground state, have been eliminated in the backresonance contribution to avoid double-counting. The 
partition function $G$ has been calculated from the many (several hundred) backresonances included in our calculations.

Fig. 1b shows the $\left(\nu_{e}, e^{-}\right)$cross sections for ${ }^{56} \mathrm{Fe}$, calculated at temperatures $T=10^{10}$ $\mathrm{K}, 1.5 \cdot 10^{10} \mathrm{~K}$ and $2 \cdot 10^{10} \mathrm{~K}$. In ${ }^{56} \mathrm{Fe}$ the backresonances are located at an excitation energy of $E_{i} \sim 7-9 \mathrm{MeV}$. Thus they are hardly populated at these temperatures and, as a consequence, the backresonances influence the cross section only at neutrino energies $E_{\nu} \leq 7$ $\mathrm{MeV}$. Despite the negative Q-value, the $\left(\nu_{e}, e^{-}\right)$cross section at finite temperature does not vanish at $E_{\nu}=0$. Still it remains rather small at the low neutrino energies, even at the high temperatures.

Haxton has pointed out that, after the bounce, infalling matter might get preheated by $\nu_{e}$-absorption before it is reached by the shock [4. This matter is expected to be mainly ${ }^{56} \mathrm{Fe}$. As the average $\nu_{e}$ energy for this preheating process is about $15 \mathrm{MeV}$ and the matter temperature is below $T=10^{10} \mathrm{~K}$, our calculation indicates that the relevant cross section can be calculated neglecting temperature effects. The respective cross sections are presented in [10].

During the core contraction $\nu_{e}$ absorption on nuclei is strongly hindered by Pauli blocking of the electron in the final state. In step 3, we have investigated this blocking by introducing a blocking factor $\left(1-f\left(E_{e}\right)\right)$ into Eq. (2), where $f\left(E_{e}\right)$ is a Fermi-Dirac distribution with temperature $T$ and chemical potential $\mu_{e}$. We have calculated the cross section for 3 relevant stellar conditions defined by temperature $(T$ in $\mathrm{MeV})$ and density $\left(\rho\right.$ in $\left.10^{10} \mathrm{~g} / \mathrm{cm}^{3}\right)$ : $(T, \rho)=(0.86,1),.(1.29,10$.$) , and (1.72,100$.$) . The corresponding chemical potentials, calcu-$ lated as described in [9] and assuming the same electron-to-baryon ratios $Y_{e}$ as in Fig. 1 of [5] at these densities, are 8.3 MeV, 18.1 MeV and 36.2 MeV, respectively. The blocked cross sections are shown in Fig. 1c. We note that, for absorption on backresonances, the electron in the final state additionally gains the nuclear excitation energy in the initial state and hence is less affected by the blocking. Obviously the importance of the backresonances is a competition of the Boltzmann weight in the thermal ensemble, which increases with temperature, and the Pauli blocking, which decreases the cross section. As the chemical potential, which sets the scale for the blocking, increases faster than the temperature the blocking becomes dominant with increasing temperature and density. Although the cross sections are still finite at low neutrino energies and are clearly enhanced compared to calculations performed at $T=0$, i.e. for the ground state only, the respective cross section values are significantly smaller than the competing inelastic neutrino scattering of electrons and nuclei as given in [5].

The nucleus ${ }^{56} \mathrm{Fe}$ is not typical to explore finite temperature effects on neutrino absorption cross section in supernova conditions for 3 reasons. (i) The matter composition is more neutronrich, when neutrino transport is important in late stellar evolution. As a consequence, absorption of electron neutrinos has usually positive $Q$-values and does not have to overcome energy thresholds. (ii) Due to the Ikeda sumrule the GT strength in the $\left(\nu_{e}, e^{-}\right)$ direction increases proportional to the neutron excess $(N-Z)$, while the backresonance contribution is reduced due to blocking of the neutron phase space in the final state. This effect enlarges the relative importance of the ground state contribution in Eq (2) with respect to the backresonances. However, there is an interesting third point related to nuclear structure. (iii) The energy position of the backresonances depends on the pairing structure of the nucleus [9]. In odd-odd nuclei the backresonances reside at significantly lower excitation 
energies $\left(E_{i} \sim 1-3 \mathrm{MeV}\right)$ than in odd- $A$ nuclei $\left(E_{i} \sim 4-6 \mathrm{MeV}\right)$, while they are largest in even-even nuclei. To explore the influence of these 3 effects on the finite-temperature neutrino absorption cross sections we have performed calculations, similar to those discussed for ${ }^{56} \mathrm{Fe}$ in Figs. $1 \mathrm{~b}$ and 1c, for the two even-even nuclei ${ }^{60,62} \mathrm{Ni}$, the odd- $A$ nuclei ${ }^{59,61} \mathrm{Fe}$ and the odd-odd nuclei ${ }^{60,62} \mathrm{Co}$.

Fig. 2 shows the temperature dependence of the absorption cross section for these nuclei. The even-even $\mathrm{Ni}$ isotopes still have negative $Q$-values and hence show a similar behavior as ${ }^{56} \mathrm{Fe}$. The situation is different for the other four nuclei where neutrino absorption is possible even for $E_{\nu}=0$. The influence of the Ikeda sumrule is clearly visible if comparing the cross sections for two isotopes: The enhancement due to finite temperature is reduced the more neutronrich the nucleus. This effect is strengthened by the accompanying increase in the $Q$-value. For ${ }^{60} \mathrm{Co}$ we observe an increase of the cross section at finite temperature by several orders of magnitude at low neutrino energies. This has two reasons. As mentioned above, the backresonances reside at rather low excitation energies $\left(E_{i}=1-2 \mathrm{MeV}\right)$ and are rather easily accessible by thermal excitation. However, ${ }^{60} \mathrm{Co}$ serves as an example for another nuclear structure effect which is often encountered for odd-odd nuclei. This nucleus has a ground state spin of $J=5^{+}$which introduces a strong angular momentum mismatch with the daughter nucleus and a significantly reduced effective $Q$-value for GT transitions. As a consequence a calculation of the absorption cross section for odd-odd nuclei solely on the basis of the ground state GT distribution is not a very accurate approximation at small neutrino energies. For the stellar environment this inaccuracy does not matter too much as electron blocking in the final state dominates the stellar cross sections. This is demonstrated in Fig. 3. Although the stellar cross sections are noticeably enhanced by about 2 orders of magnitude at low neutrino energies due to finite temperature effects, these cross sections are still noticeably smaller than the competing ones for inelastic neutrino scattering on electrons and nuclei. When compared to the stellar rates of these competing reactions (Fig. 1 of [5]), neutrino absorption on nuclei is important during the stellar collapse only for neutrino energies at which finite temperature effects are negligible.

How does finite temperature affect the absorption cross section of antineutrinos in the stellar environment? We have performed calculations, using Eq. (2), for the same set of nuclei as discussed above for neutrino absorption. To understand the results shown in Fig. 4 , one has to consider that some of the items listed above invert for the $\left(\bar{\nu}, e^{+}\right)$reactions. Hence, i) the $Q$ values become less favorable with increasing neutron excess and the reactions have to overcome noticeable thresholds. ii) Due to the Ikeda sumrule the contribution of the backresonances becomes increasingly more important with growing neutron excess. For neutronrich nuclei the largest contribution of the $\mathrm{GT}_{-}$strength is in the isospin $\Delta T=-1$ transition which reside at rather low excitation energies. (iii) The energy positions of these backresonances are also dependent on the pairing structure, again being lowest for odd-odd nuclei. Finally one has to consider that the chemical potential for positrons is the negative of the electron chemical potential. Thus there is no final state blocking for antineutrino absorption under core-contraction condition. We observe from Fig. 4 that finite-temperature effects significantly effect the cross sections for antineutrino energies $E_{\bar{\nu}}<10 \mathrm{MeV}$, leading to finite and sizable results even for $E_{\bar{\nu}}<Q$. We also observe that the cross sections do not depend too strongly on nuclear structure, i.e. they are roughly the same for the various nuclei at a given temperature. 
In summary, we have studied the effects of finite temperature on the stellar neutrino and antineutrino absorption cross sections. We find that the neutrino cross sections for lowenergy neutrinos are enhanced at finite temperature mainly due to the thermal population of the backresonances. However, the electron chemical potential in the supernova environment increases more rapidly than the temperature. Thus the stellar absorption rates for lowenergy neutrinos are dominated by electron blocking in the final state making the stellar neutrino absorption rate much smaller than the competing inelastic neutrino scattering off electrons and nuclei. Neutrino absorption might be important during the stellar collapse for higher neutrino energies where temperature effects are unimportant. Furthermore an accurate description of the GT distribution is probably not required at these higher neutrino energies and the relevant rates can be obtained on the basis of less sophisticated nuclear models, like the random phase approxmation. These calculations, which are in progress, have to include transitions mediated by higher multipole operators using also the correct dependence of these operators on momentum transfer.

Antineutrino absorption cross sections on neutronrich nuclei are strongly enhanced at finite temperature for small antineutrino energies. As the stellar rates are roughly the same for different nuclei it might be sufficient to represent this process by a typical nucleus in core-collapse simulations.

We like to end with a remark on finite temperature effects on inelastic neutrino scattering on nuclei. This process (together with inelastic scattering on electrons) is considered as the mechanism to downscatter neutrinos in energy where they can escape from the star more easily. However, at finite temperature inelastic neutrino scattering off nuclei will also be enhanced, mainly due to the thermal population of the backresonances in the $\mathrm{GT}_{0}$ distribution. These backresonance contributions will, however, increase the neutrino energies. We are currently investigating the relevance of this effect in more details.

\section{ACKNOWLEDGMENTS}

Our work was supported in part by the Danish Research Council and by the Swiss National Science Foundation. 


\section{FIGURES}

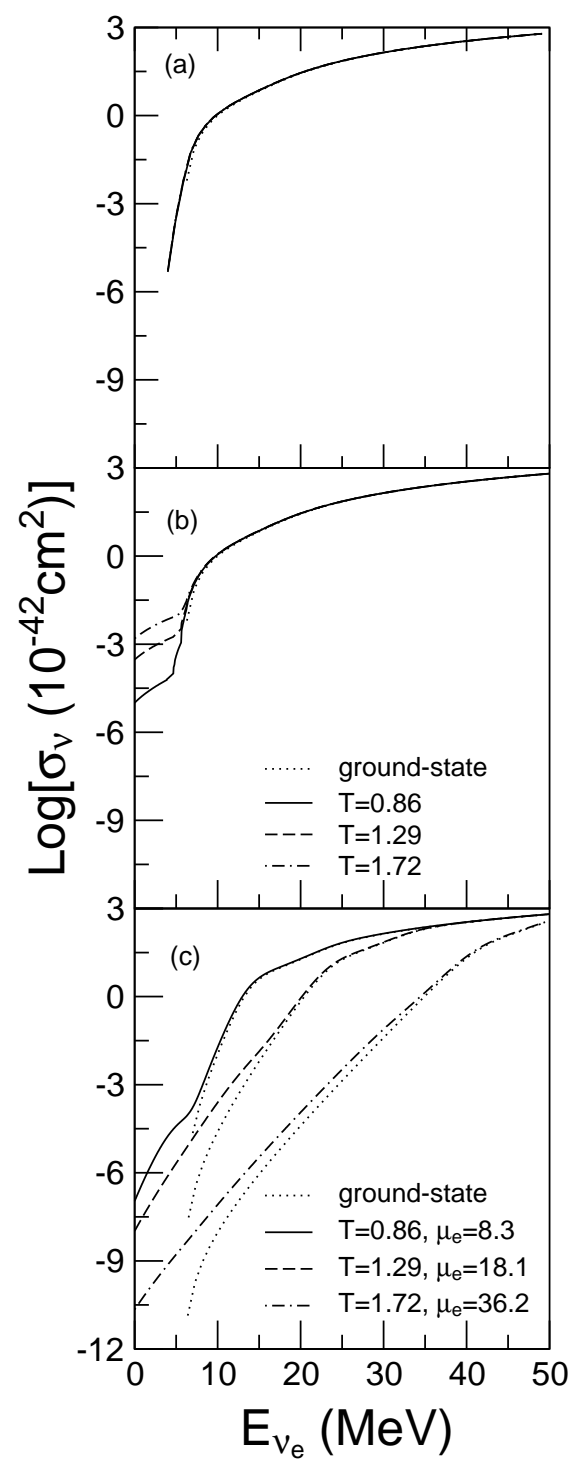

FIG. 1. Absorption cross section of electron neutrinos on ${ }^{56} \mathrm{Fe}$. a) Comparison of the cross section calculated for the ground state (dotted) with the one obtained at finite temperature $T=0.86$ $\mathrm{MeV}$. The calculations have been performed using Eq. (1) where the 4 lowest states in ${ }^{56} \mathrm{Fe}$ have been considered in the thermal ensemble. b) Comparison of the cross section calculated at finite temperatures (in $\mathrm{MeV}$ ) with the one derived from the ground state only (dotted). The calculations at finite temperatures have been performed using Eq. (2) without consideration of electron blocking in the final state; c) the same cross sections as in b) but now considering the electron blocking in the final state. The respective temperatures and chemical potentials (both in $\mathrm{MeV}$ ) are given in the figure. 


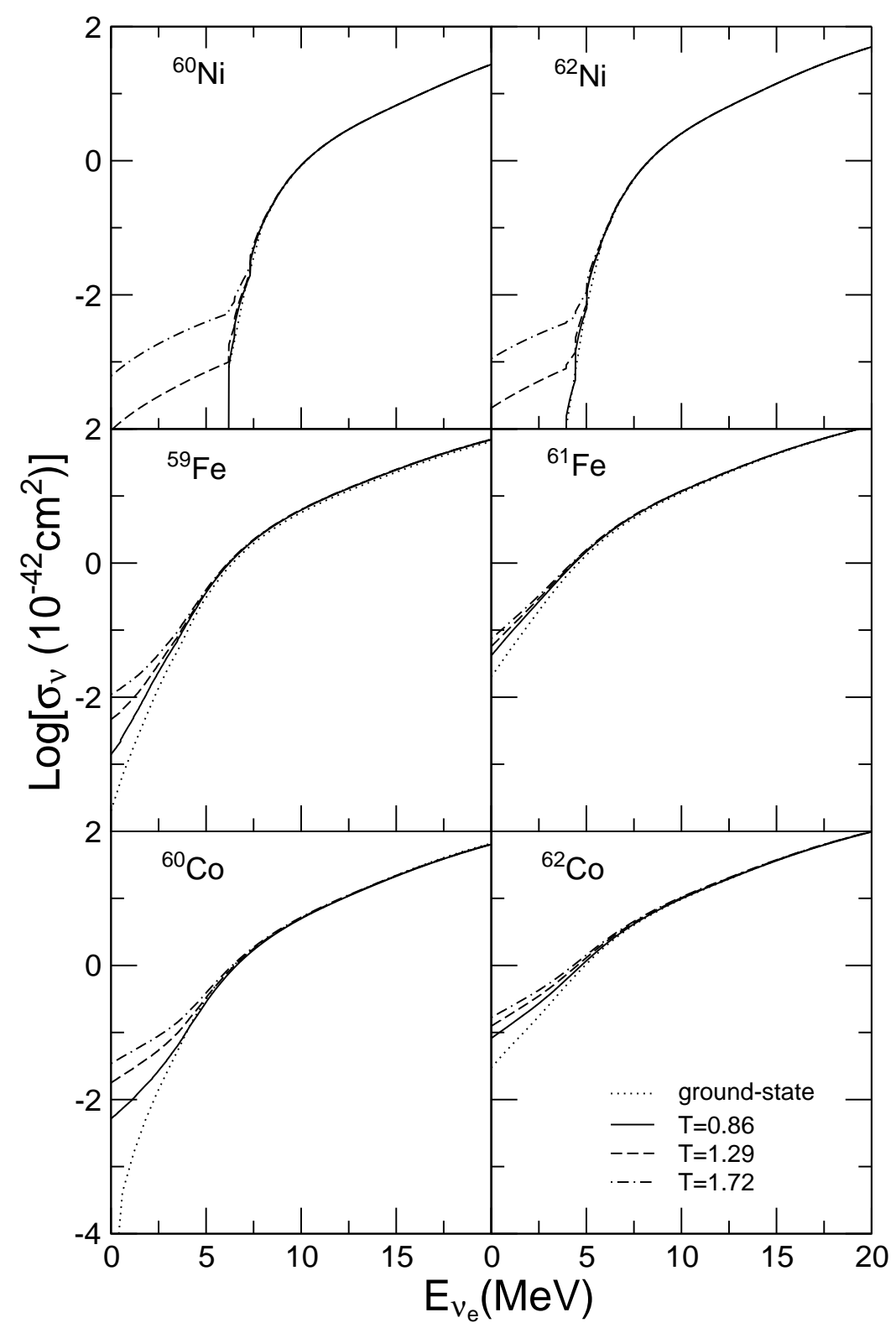

FIG. 2. Absorption cross section of electron neutrinos on selected neutronrich nuclei. The calculations have been performed for the same temperatures as in Fig. 1b) (temperature defined in $\mathrm{MeV}$ in the figure) using Eq. (2). The finite-temperature results are compared to the one derived from the ground state (dotted). 


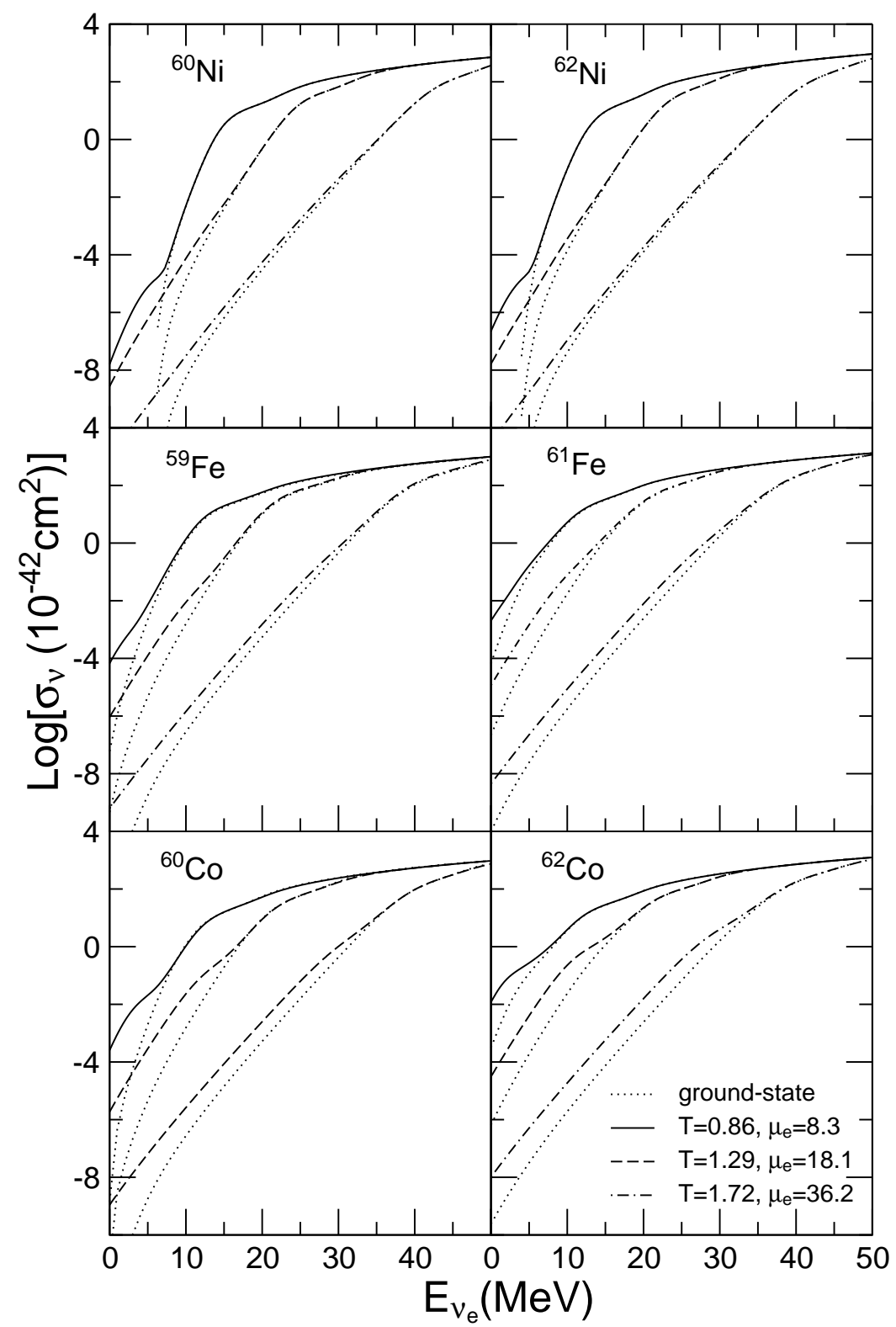

FIG. 3. The same as Fig. 2, but now including the Pauli blocking of the electron in the final state. The stellar conditions are defined by the temperature and the chemical potential (both in $\mathrm{MeV}$ ). The finite-temperature cross sections are compared to the one derived from the ground state alone. 


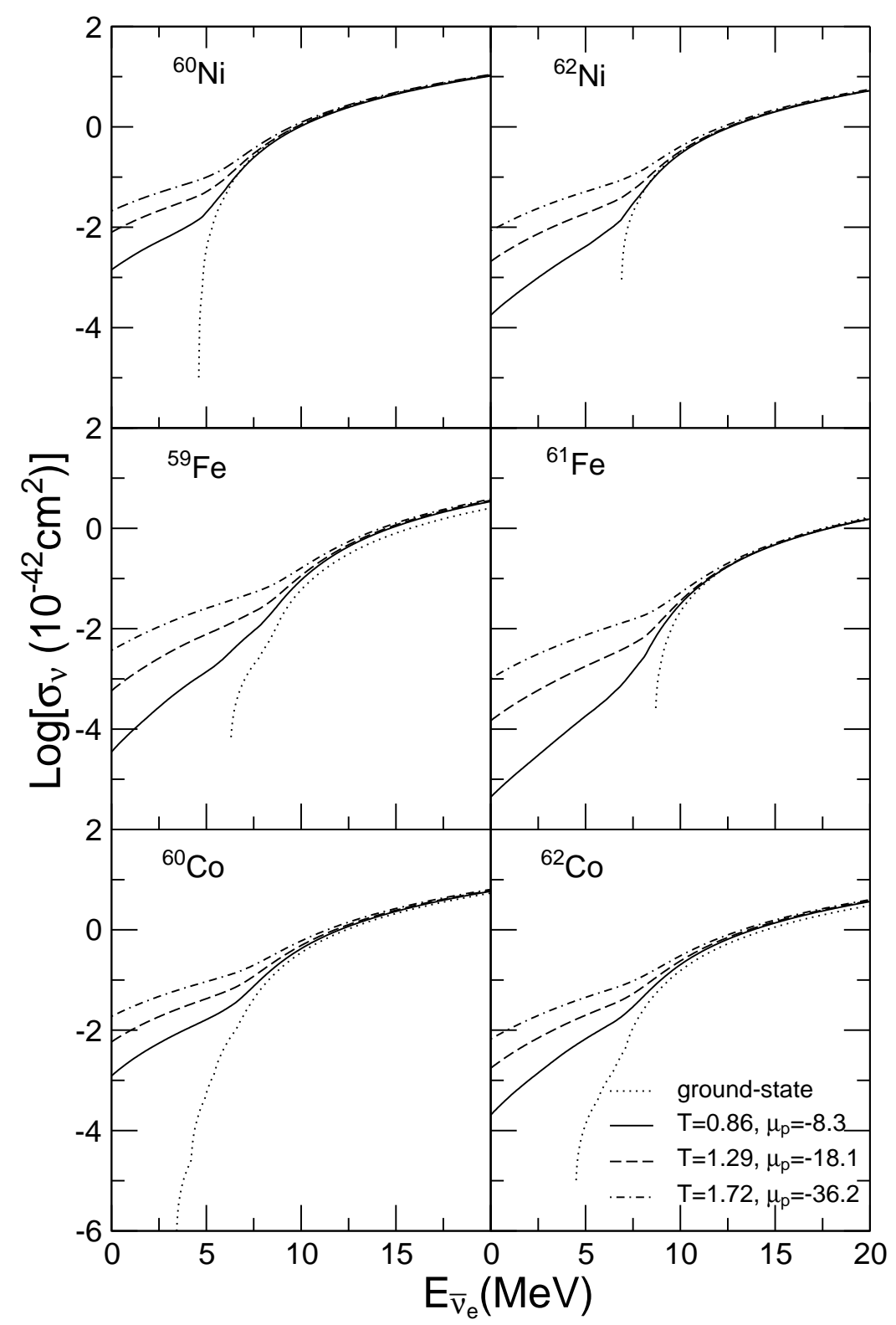

FIG. 4. The same as Fig. 2, but for antineutrinos. Final state blocking for positrons is negligible for the chosen core collapse conditions. The backresonance contribution also includes the one for the Fermi transition, which, however, is at too high an excitation energy to influence the cross sections. 


\section{REFERENCES}

[1] H. A. Bethe, Rev. Mod. Phys. 62 (1990) 801;

[2] S.W. Bruenn, ApJS 58 (1985) 771

[3] A. Mezzacappa and S. W. Bruenn, Ap. J. 410 (1993) 740; A. Mezzacappa et al., Phys. Rev. Lett. 86 (2001) 1935

[4] W. C. Haxton, Phys. Rev. Lett. 60 (1988) 1999.

[5] S.W. Bruenn and W.C. Haxton, ApJ. 376 (1991) 678

[6] M.B. Aufderheide, I. Fushiki, S.E. Woosley and D.H. Hartmann, ApJS 91 (1994) 389

[7] G.M. Fuller, W.A. Fowler and M.J. Newman, ApJS 48 (1982) 279

[8] E. Caurier, K. Langanke, G. Martínez-Pinedo and F. Nowacki, Nucl. Phys. A653 (1999) 439.

[9] K. Langanke and G. Martínez-Pinedo, Nucl. Phys. A673 (2000) 481

[10] J. Toivanen et al., submitted to Nucl. Phys. A 